PHYSICAL REVIEW E 86, 011306 (2012)

\title{
Transition dynamics and magic-number-like behavior of frictional granular clusters
}

\author{
Antoinette Tordesillas* and David M. Walker \\ Department of Mathematics and Statistics, University of Melbourne, Parkville, Victoria 3010, Australia \\ Gary Froyland \\ School of Mathematics and Statistics, University of New South Wales, Sydney, New South Wales 2052, Australia \\ Jie Zhang \\ Department of Physics, Shanghai Jiao Tong University, Shanghai 200240, China \\ Robert P. Behringer \\ Department of Physics, Duke University, Durham, North Carolina 27708, USA \\ (Received 20 February 2012; published 30 July 2012)
}

\begin{abstract}
Force chains, the primary load-bearing structures in dense granular materials, rearrange in response to applied stresses and strains. These self-organized grain columns rely on contacts from weakly stressed grains for lateral support to maintain and find new stable states. However, the dynamics associated with the regulation of the topology of contacts and strong versus weak forces through such contacts remains unclear. This study of local self-organization of frictional particles in a deforming dense granular material exploits a transition matrix to quantify preferred conformations and the most likely conformational transitions. It reveals that favored cluster conformations reside in distinct stability states, reminiscent of "magic numbers" for molecular clusters. To support axial loads, force chains typically reside in more stable states of the stability landscape, preferring stabilizing trusslike, three-cycle contact triangular topologies with neighboring grains. The most likely conformational transitions during force chain failure by buckling correspond to rearrangements among, or loss of, contacts which break the three-cycle topology.
\end{abstract}

DOI: 10.1103/PhysRevE.86.011306

PACS number(s): 81.05.Rm

\section{INTRODUCTION}

Modern materials science abounds with explorations into a diverse array of dissipative media (e.g., concentrated emulsions, granular materials, cellular materials, slurries, pastes, and molecular glasses) [1-8]. In part, this broad appeal arises from dissipative media's distinctive mechanical response to applied forces. On a macroscopic scale, such response often bears the hallmarks of complexity, including self-organization and emergent behavior (e.g., [2-5,9-12]). By far the best examples of these patterns of behavior, common in both nature and technology, are those exhibited by dense granular materials under quasistatic loading conditions [10-19]. Here we study the underlying mesoscopic mechanisms for such behavior, paying particular attention to recurring ordered arrangements (i.e., structural motifs) and preferential rearrangements which are larger in length scale than the constituent grains. Unravelling the details of these mesoscopic structures and associated frictional rearrangements, and their impact on bulk mechanical response-particularly on the development of failure-presents a great scientific challenge [7,18,20,21]. Indeed, although granular materials have stimulated intense research activity for decades [5-8], robust predictions of their mechanical behavior under load remain elusive [12,22-26]. To date, a crucial missing element that limits prediction and control of dense granular behavior, particularly that of inelastic deformation and failure, is a precise understanding of the interplay between the dynamics of prevalent and

\footnotetext{
*Corresponding author: atordesi@ms.unimelb.edu.au
}

persistent particle rearrangements and the evolution to failure of key functional mesoscopic structures, epitomized by major load-bearing force chains $[10,14,18,26-32])$. Knowledge of the dynamical rules governing these essential inelastic rearrangements, especially as they relate to the stability of and energy released from force chains, is crucial for reliable model predictions [11,21]. Advances in this respect hold great promise for not only the control of granular behavior in many practical settings (e.g., forecasting and mitigation of earthquakes and landslides [17,31,32], improvement of road and off-road transport infrastructure [33], development of mining processes and technologies which minimize environmental impact and energy use [7,8,34]) but also the creation of novel materials with levels of functionality similar, if not potentially superior, to those observed in nature (e.g., bio-inspired ceramics) [1].

Recent numerical and experimental developments have yielded insights into how the local structural behavior of columnar force chains leads to inelastic deformation and failure at the macroscopic scale [9-18,21,30,31,35-41]. As the material deforms in response to applied stresses, the constraints imposed by the local structure influence the resultant kinematics of individual grains, both in these strong columns and their relatively weak confining neighbors. Experiments and discrete element (DEM) simulations have shown that conformational rearrangements occur frequently, transitioning the system towards the various stable structural configurations accessible to the system (e.g., $[11,42,43])$. These dissipative rearrangements, resisted by interparticle contact friction and geometric particle interlocking, are strongly nonlinear and heterogeneous in space and time (e.g., [11-13,15,21,44]). 
The most pronounced manifestations of these rearrangements occur during failure in the presence of shear bands (e.g., [13,15,17,31,41,44]). A shear band is a region in the material in which deformation and particle rotations are concentrated. Once fully developed, shear bands split the granular body into parts which may move relative to each other in near-rigid body motion [9,15,44,45]. Grain-scale information from noninvasive micromechanical experiments on sand and photoelastic disk assemblies suggests that force chains fail by buckling and that their collective localized buckling is the mechanism underpinning shear band formation and, ultimately, macroscopic localized failure [13,37,44]. A comprehensive experimental and numerical investigation into force chain buckling in a series of monotonic and cyclic shear tests, for a range of granular materials, corroborates this [11,21,30,36-41]. Moreover, cooperative evolution among linear and cyclic building blocks of self-organization (i.e., force chains and surrounding minimal contact cycles) has been observed $[11,16,39,40]$. How force chains regulate their contacts with respect to both topology and the forces they carry for a given loading condition is largely unknown. An overarching question is whether particles rearrange in response to the changing applied forces to provide optimum stability to these axially loaded columns of grains. Transition pathways between granular cluster conformations in a stability landscape would provide such information, but to date, these have not been established for any granular system. Here we report on a study of these transition pathways from the standpoint of cluster topology and structural stability. By probing relative probabilities of cluster conformations and conformational transitions we find that there are favored conformations residing in distinct structural stability states. Analysis of stability transitions and associated dynamical barriers elucidates the role of topology in the stability and failure of force chains by buckling.

The rest of this paper is arranged as follows. In Sec. II, we provide details of the experimental system. Next, we present the Markov transition matrix analysis of the granular system under cyclic shear in Sec. III. A Markov transition matrix analysis of the dynamics of conformational transitions, with special attention paid to force chains, is given in Sec. IV. In Sec. V, the structural stability landscape of cluster conformations is examined, with the transitions in conformational stabilities similarly quantified using a Markov transition matrix. The stability of force chains, and the stability transitions specific to the failure of force chains by buckling, is presented. We close the paper in Sec. VI with a discussion of the implications of the work for constitutive modeling of dense granular media, and highlight some common ground and potential synergies with studies of molecular complex systems.

\section{THE CYCLIC SHEAR EXPERIMENT: SYSTEM AND METHOD}

The system comprises a bidisperse, densely packed assembly of frictional circular disks subject to quasistatic cyclic shear under constant volume (Fig. 1). The experiment involves 1568 bidisperse disks (particles), confined in a biaxial apparatus: $\sim 80 \%$ have diameter $0.74 \mathrm{~cm}$ and $\sim 20 \%$ have diameter $0.86 \mathrm{~cm}$. The particles rest on a flat, smooth, horizontal surface,

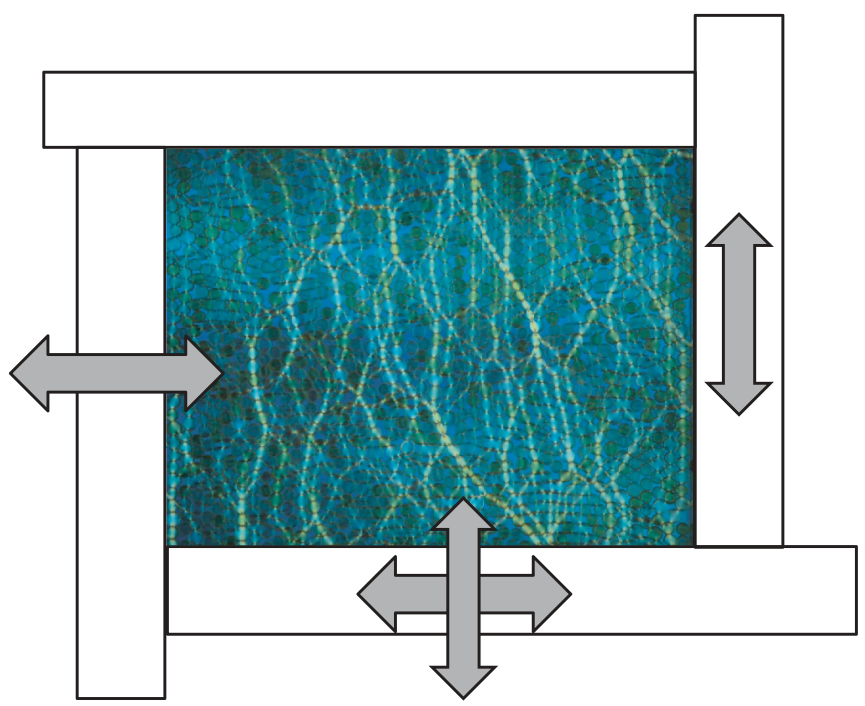

FIG. 1. (Color online) A sketch of the boundary walls of the pure shear experimental setup, with the top wall fixed and the arrows in the remaining three walls indicating their allowable motions. The assembly of circular photoelastic disks occupy the rectangular area bounded by the four walls.

and the walls of the Biax move to create specific strains, here pure shear: compression in one direction, dilation in the other, with fixed system area and density.

The initial state is specified by square system boundaries, a (fixed) packing fraction of $\phi=0.795$, and as nearly isotropic and stress-free conditions as possible. We then shear the system cyclically, first by compressing (expanding) in the vertical (horizontal) directions, keeping the area fixed. After reaching a maximum shear deformation, we reverse the shear strain, so that there is compression (expansion) along the horizontal (vertical) axes. We carry out reverse shear past the configuration of square boundaries, until the shear strain reaches a minimum negative value. We again reverse the shear strain, and we return the boundaries to a square. This completes one shear cycle. Starting from the final state of the first shear cycle, we then apply similar cyclic strains for a total of six shear cycles. Here we examine the data for only the first two shear cycles; subsequent shear cycles exhibit the same dynamics, as established earlier in Ref. [41]. Note that a shear band develops at certain stages of each shear cycle.

Each step in the quasistatic cyclic shear consists of a small increment of strain $(0.3 \%)$. After each small step, we freeze the boundaries and acquire three images: one with polarizers, one without polarizers, and a third that allows us to track disk position, rotation, and displacement. The disks are made of a photoelastic material which is birefringent under an applied strain. The image of a photoelastic particle, placed between crossed polarizers, shows light and dark bands that are determined by the stresses within the disk (Fig. 2 top left). These stresses are in turn fixed by the (vector) contact forces acting on the particle. We solve the inverse problem for each photoelastic disk image to obtain the interparticle contact forces $[41,46]$. The photoelastic particles are frictional. We consider the particles in a cluster to interact through their contacts via contact forces and contact moments that are governed by (i) an elastic normal force given by Hooke's 


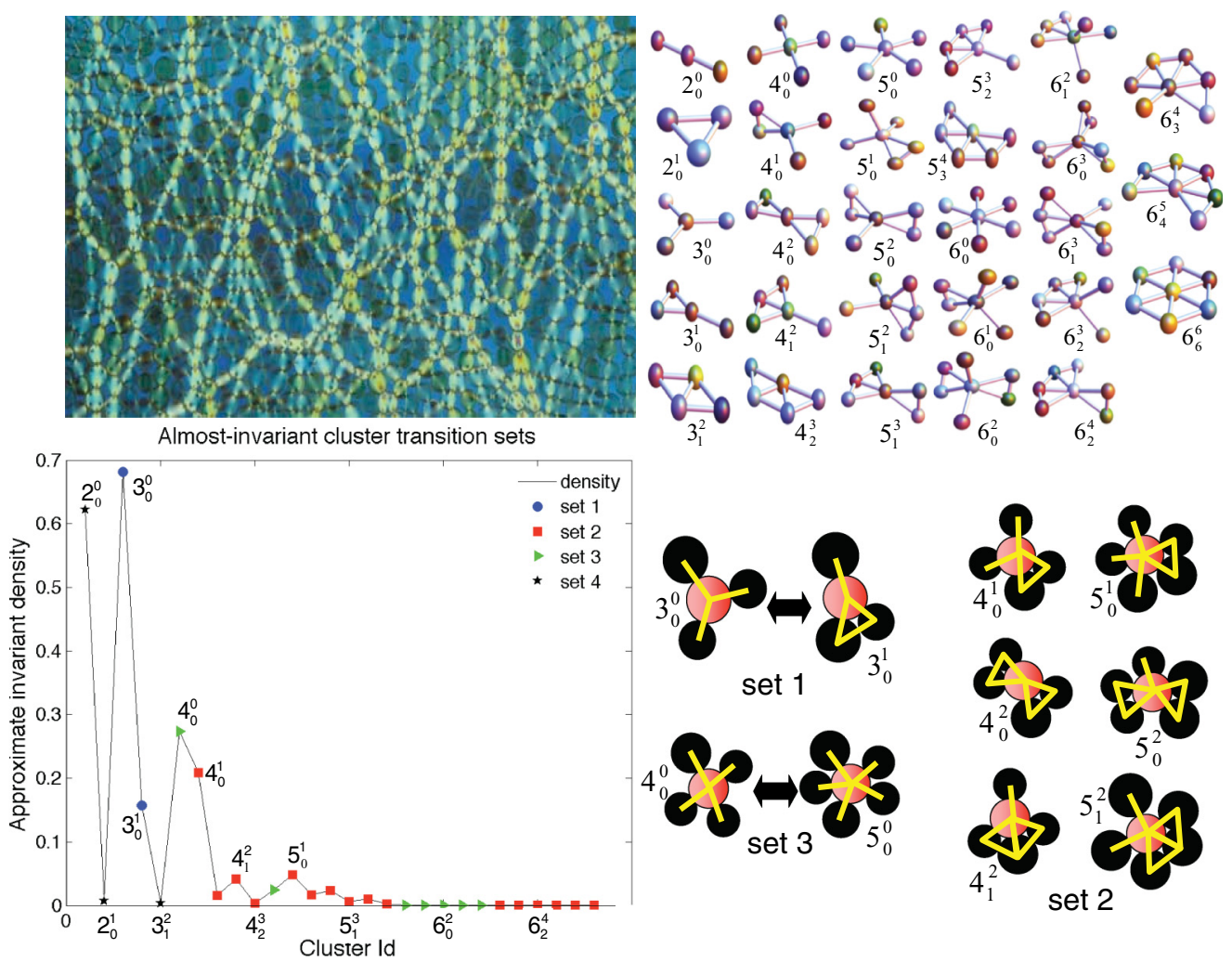

FIG. 2. (Color online) Granular cluster conformations. Image of the assembly of photoelastic birefringent circular disks showing the force chains when sample is compressed vertically (top left). Subgraphs of the 28 conformations found for the experimental system throughout loading history (top right). Densities of conformations shown as solid line, and the most prevalent clusters classified into four almost-invariant sets distinguished by color and symbol (bottom left). Representative members of three of these sets are drawn as clusters, showing that typical rearrangements are due to a single contact forming or breaking for the same number of disks or a new disk joining the cluster (bottom right).

law (spring), (ii) an elastic or plastic tangential force given by Hooke's law if elastic and Coulomb's friction law if plastic (spring slider), and (iii) an elastic or plastic contact moment analogous in form to the tangential force [11].

In summary, after each step or strain state of the cyclic shear, we have information on all the particles' positions, contacts, and contact forces. The particles' elastic stiffnesses corresponding to the normal and tangential contact forces and the contact moment, as well as the sliding and rolling friction coefficients, are all known, and we refer readers to Ref. [11] for complete details of the procedure used to obtain these quantities. Altogether this set information enables us to characterize various aspects of the contact topology, as well as compute the structural stability, of each particle and particle cluster of any size at each strain state of the loading history [11].

\section{MARKOV TRANSITION MATRIX ANALYSIS}

The evolving mechanical response of the granular assembly is quantitatively characterized by analysis of the individual equilibrium states, each corresponding to a strain state in the loading history, and their sequential state-to-state transitions. The contact topology and structural stability of cluster conformations define the states of the granular assembly, and the resultant dynamics of the system under cyclic shear is assumed to be close to stationary.
A Markov transition matrix $P$ is constructed by counting the observed transitions that each cluster conformation undergoes over the many time steps throughout loading history. The states of the transition matrix are either (i) the granular cluster conformation or (ii) the interval of stability in which a granular cluster's structural stability sits. The matrix $P$ is normalized to be a row stochastic matrix, and the entries of the leading left eigenvector give an approximation to the invariant probability measure of the assumed underlying stochastic process modeling the dynamics. The approximate invariant measure and $P$ can be used to construct a time-reversible Markov matrix $R$ whose eigenspectrum, starting with the second largest eigenvalue, can be used to determine almostinvariant transition sets [47]. Gaps in the spectrum of real eigenvalues of $P$ indicate how many eigenvectors of $R$ to use in order to find a given number of almost-invariant transition sets. The eigenvalue separation can also be used to gauge whether the time-step interval length is appropriate.

The analysis in Ref. [47] is formally valid if the stochastic process on the states (here either cluster confirmations or stability intervals) is stationary. In practice, the checking of stationarity with a finite-duration data set is impossible. Nevertheless, the analysis of Ref. [47] still yields powerful results in situations where this stationarity assumption is violated. For example, a series of studies of the Southern Ocean [48-50] used the same transition matrix techniques to map major oceanic gyres off the Antarctic coast. The underlying dynamics 
of the ocean is nonstationary over the two- to three-month period considered, but this period is not long enough to create highly nonstationary effects. The transition matrix approach produced surface maps of $10 \%$ greater accuracy than existing oceanographic methods and remains the only approach that enables full three-dimensional mapping. In situations where the underlying dynamics is highly nonstationary, analogous methods have recently been developed [51] and applied both to atmospheric data to map stratospheric vortices [51,52] and to ocean data to track oceanic eddies [53]. In the present setting, we believe that both the conformation and stability interval data are approximately stationary based on earlier findings from Ref. [41] that showed the statistical properties of the system (i.e., average coordination number, pressure, fabric orientation angle, etc.) are essentially constant across the shear cycles; therefore the techniques in Ref. [47] are valid. A clustering algorithm is employed to determine the (transition-state) membership of each almost-invariant set by minimizing a weighted cost function, where the weighting is closely related to the approximate invariant probability of each state [47].

\section{MESOSCALE CONFORMATIONS AND CONFORMATIONAL TRANSITIONS OF GRANULAR SELF-ORGANIZATION}

For each equilibrium state, an unweighted and undirected graph, or complex network, represents the granular assembly with the particles as the nodes, and their contacts as the connecting links or edges between corresponding nodes [35, 39,40]. Particle rearrangements are primarily responsible for the material's deformation. These are reflected in the evolving complex network of contacts: old network connections break as contacts are lost, while new connections form as contacts are created. In this study, we focus on that class of mesoscopic cluster conformations comprising a particle and its first ring of contacting neighbors (analogous to the first coordination shell in molecular clusters). This class comprises the smallest clusters for which emergent preferential orientations in contacts and contact forces can be comprehensively probed. Next we sort the clusters according to the network properties of degree (number of contacts of the central reference particle) and number of three-cycle topology (triangles) within the cluster $[35,39,54]$. The physical and geometrical restrictions imposed by the particle-size distribution and the system boundaries imply a finite number of distinct cluster conformations involving one node (particle) and its connected neighbors. We found 28 distinct cluster conformations after examining the first ring of contacting neighbors of each particle at every state throughout loading. Figure 2, top right, shows the subgraphs of the conformations, each labeled $x_{j}^{i}: x$ is the central particle degree, $i$ is the number of three-cycle topology, and $j$ is the number of shared edges among these three-cycle topology.

The transitions between the 28 cluster conformations are modeled as a discrete time Markov process [47] with one time step representing an interval of four quasistatic equilibrium states, that is, the number of times a particle of a given conformation transforms to another conformation, possibly of the same topology, after four time steps (recall Markov transition matrix analysis for an explanation on how to select a suitable time-step interval). The corresponding $28 \times 28$ transition matrix is analyzed to extract the most prevalent conformations and to identify sets of conformations which are almost invariant under the dynamics, that is, the most persistent sets of conformations [47]. In order of decreasing frequency, the most recurrent granular conformations are $3_{0}^{0}$, $2_{0}^{0}, 4_{0}^{0}, 4_{0}^{1}$, and $3_{0}^{1}$ (Fig. 2, bottom left). Almost-invariant transition sets of granular conformations are identified from the eigenvectors of the transition matrices. The number of sets are determined by the separation of the spectrum of eigenvalues using a clustering algorithm [47]; Fig. 2, bottom left, shows four almost-invariant sets found using three eigenvectors. Conformations of similar degree typically lie in the same set, with the distinction between members of each set being mainly due to the rearrangements of the first ring neighbors (i.e., no loss or gain of contacts); see Fig. 2, bottom right. The top five conditional transition probabilities $p\left(x_{j}^{i} \rightarrow y_{l}^{k}\right)$ quantifying conformational transitions from $x_{j}^{i}$ to $y_{l}^{k}$ are $p\left(2_{0}^{0} \rightarrow 2_{0}^{0}\right)=0.1771, p\left(3_{0}^{0} \rightarrow 3_{0}^{0}\right)=0.1696, p\left(2_{0}^{0} \rightarrow 3_{0}^{0}\right)=$ $0.0708, p\left(3_{0}^{0} \rightarrow 2_{0}^{0}\right)=0.0689$, and $p\left(4_{0}^{0} \rightarrow 4_{0}^{0}\right)=0.0589$.

\section{A. Topology and dynamics of force chains}

The evolution of force chains, from growth to failure, has long been held as the pivotal element of granular material strength and failure [13,14,17,27,30,31,37,44]. Specifically, the strength of a granular assembly under load (i.e., its resistance to particle rearrangements or deformation) comes from two sources. One is inherent (e.g., initial packing, particle shape, and surface properties); the other is induced by the loading conditions (i.e., by self-organization). Recent evidence of cooperative behavior among emergent mesoscopic structures, such as the formation of trusslike three-cycle topology around force chains, provides incisive clues for functional activity with governing design principles resembling those employed in architectural structures [11,16,39,40]. Given the significance of force chain evolution in the present study, we briefly delineate the key elements of the methods used to identify force chains and their failure by buckling, before proceeding with the discussion of our results (full details are provided elsewhere, e.g., [11,30,37]).

The method for identifying which particles are in force chains for a given strain state of the loading history is centered around a so-called particle load vector. This vector is computed for each particle in the assembly. It is derived from the local force moment tensor $\hat{\sigma}_{i j}$ :

$$
\hat{\sigma}_{i j}=\sum_{c=1}^{\alpha} f_{i}^{c} r_{j}^{c},
$$

where $\alpha$ is the number of contacting neighbors of the particle (or degree for the associated node in the contact network), and $f_{i}^{c}$ and $r_{j}^{c}$ denote the components of the contact force and the unit normal vector from the center of the particle to the point of contact. The largest eigenvalue of this tensor and its corresponding eigenvector define, respectively, the magnitude and direction of the particle load vector. A force chain is then defined as a quasilinear chain of three or more particles whose particle load magnitudes each exceed the global average 
value and whose particle load vectors align within a prescribed small tolerance angle (here, we choose $45^{\circ}$ ). The direction of alignment of the particle load vectors of the constituent force chain members determines the trajectory of force transmission through the chain.

In contrast to force chains, which are established for individual strain states of the loading history, the method for identifying which parts of the force chain particle network have undergone buckling, that is, buckled force chain (BFC) segments, applies to intervals or increments of strain. When a force chain buckles from axial compression, the axial load it carries decreases as its constituent particles displace laterally from the chain's initial longitudinal configuration. Accordingly, for a given strain interval $\left[\varepsilon^{A}, \varepsilon^{B}\right]$, the method is a three-step process of elimination: step 1, eliminate all particles that are not in force chains at $\varepsilon^{A}$; step 2 , of the force chains left from step 1, eliminate those whose member particles do not individually sustain a drop in the magnitude of their particle load vector during $\left[\varepsilon^{A}, \varepsilon^{B}\right]$; and step 3 , of the force chains left from step 2, compute the change in alignment of all contiguous three-particle segments in terms of the buckling angle, $\theta: \theta=\left(\theta^{A}-\theta^{B}\right) / 2,0<\theta^{B}<\theta^{A} \leqslant \pi$, where $\theta^{A}$ and $\theta^{B}$ are the subtended angle of the outer particles with respect to the central particle in the segment at strain states $\varepsilon^{A}$ and $\varepsilon^{B}$, respectively. A force chain segment, as well as the force chain of which it is part, is deemed to have buckled (i.e., a BFC) if the buckling angle exceeds a prescribed threshold $\theta^{*}$, that is, $\theta^{*}<\theta$. Using data from DEM simulations and photoelastic disk experiments, the sensitivity of the results from these methods to the various thresholds adopted for identifying force chains and BFCs has been studied extensively elsewhere (e.g., [11,30,37,39]). The threshold values of global average particle load vector magnitude for the force chain algorithm along with $\theta^{*}=1^{\circ}$ for the buckling algorithm are physically reasonable choices.

We now turn our attention to the topology and dynamics of cluster conformations for force chain particles. First, consistent with past observations $[14,27,44]$, it can be seen from Fig. 3 that force chains typically align in the direction of most compressive principal stress. As such, force chains are axially compressed. To function effectively as load-bearing columns, these must be supported laterally through contacts with comparatively weak neighboring particles [30]. Notice the stark contrast in the brightness of the birefringence patterns between the particles in the central force chain column and their surrounding neighbors in Fig. 3. At any given strain state of the cyclic shear experiment, force chain particles comprise around $10 \%$ to $60 \%$ of the total number of particles corresponding to unjammed and jammed states, respectively $[11,41]$. We find these force chains reside in regions of relatively high connectivity, as evident in their higher average degree and clustering coefficient (a measure of the density of three-cycle configurations in a network [16,40]); see, for example, the relatively higher degree of member particles in the initial stages 1-3 compared to the last stages 9 and 10 when the force chain has buckled in Fig. 4. Past studies have revealed strong similarities in force chain behavior for a wide range of material properties and boundary conditions [11,30,38,40,41]. For example, here we find the previously observed trend that force chains favor contacts with laterally supporting
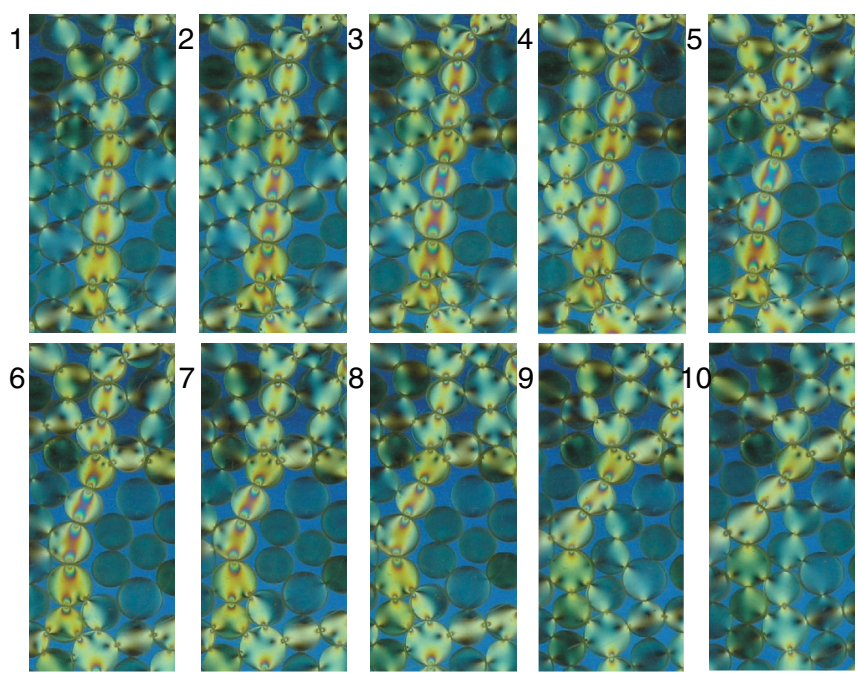

FIG. 3. (Color online) The evolution of the network topology of contacts and contact forces for a representative force chain over 10 consecutive stages of the loading history, during which the most compressive principal stress at the macroscopic scale is aligned in the vertical direction. Force chain evolution is driven by cooperative interactions among structural building blocks. Columnar force chains bearing the majority of the load rely on confining contacts with comparatively weak neighbors for lateral support. As the force chain buckles, some three-cycle topology strengthen, leading to three-force cycles [39], which prop up the buckling chain to restore alignment. Compare, for example, stage 1 with stages 6 and 7: Note the increased birefringence brightness from the left (right) three-cycle supporting the second and third (first, fifth, and sixth) particles from the bottom of the force chain identified in Fig. 4.

neighbors in three-cycle, trusslike formations [11,39], specifically, through conformation $4_{0}^{1}$ (Figs. 3 and 4). Nonforce chains frequently, though not exclusively, comprise particles in less connected conformations $2_{0}^{0}$ and $3_{0}^{0}$, which have no three-cycle topology. A dual buckling resistance underpins this cooperative evolution between columnar force chains and trusslike three-cycle topology: Three-cycle topology (i) "prop up" the force chain to restore alignment (Figs. 3 and 4) and (ii) frustrate particle rotations integral to buckling [16,39].

Force chains are not only primary load bearers but are also major repositories of stored energy in the system [19,27,29,38]. Consequently, their failure by buckling directly (i) reduces the load the material can carry and (ii) leads to dissipation following the collective release of stored energy accumulated at member contacts [30,37,38]. For instance, note the decrease in the brightness of the birefringence pattern in Fig. 3 as the force chain buckles. Load-carrying capacity and energy dissipation form the two halves of mathematical formulations that relate the deformation to the stress in a dissipative material (i.e., continuum models). To achieve reliable and robust predictions, such models must incorporate the kinematics of those rearrangement events which are chiefly responsible for energy dissipation (i.e., internal variables or plastic strains [20,23,26,36]). Knowledge of which conformational transitions are likely to occur when a force chain buckles and their associated probabilities is an essential and heretofore missing element in continuum models [26,30]. To date, the only recourse has been to offer either an "educated 
1
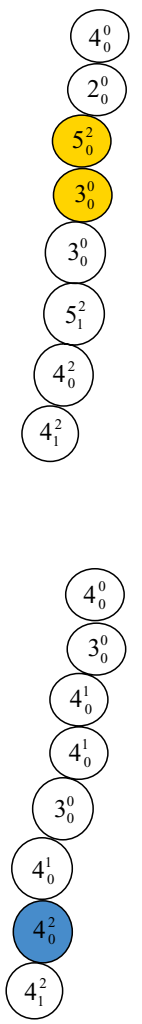

2

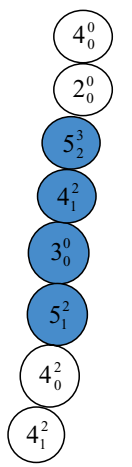

7

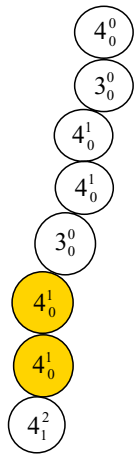

3

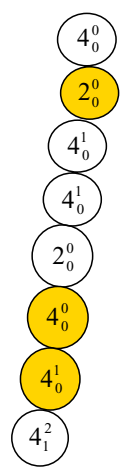

8

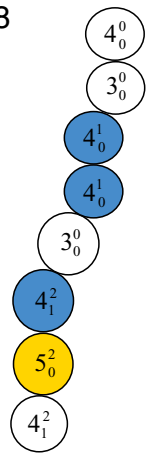

4
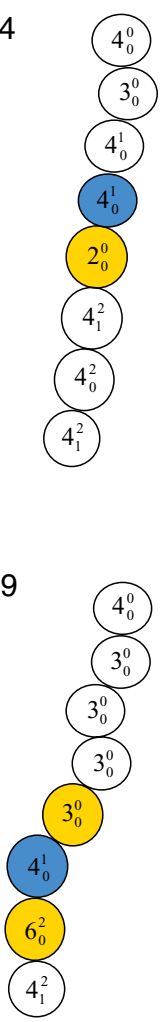

5

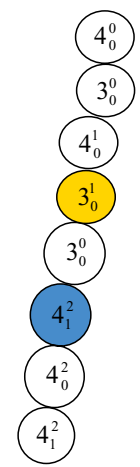

FIG. 4. (Color online) The conformational transitions of the particles in the force chain in Fig. 3. Particles which change in conformation by the next stage, over stages 1-9, are colored: light yellow (light gray) particles gain connectivity by changing contacts and/or three-cycle topology, whereas dark blue (dark gray) particles lose connectivity. Together with Fig. 3, this figure highlights the dynamics of particles in force chains associated with the continual regulation of the topology of, and forces through, their contacts.

guess" [26,30] or empirical evolution laws for the plastic strain with no clear connection to the geometric or mechanical details associated with force chain buckling [20,22,29]. This study shows that, within a buckling force chain, the most likely conformational transitions tend to either preserve the topology or involve a transition from a more connected conformation (i.e., $3_{0}^{1}$ or $4_{0}^{1}$ ) to a less connected conformation (i.e., $3_{0}^{0}$ ), as seen in Figs. 3 and 4. Conformational transitions from $4_{0}^{1}$ to $3_{0}^{0}$ involve loss of both a contact and supporting three-cycle. Such transitions are not the most dominant within the assembly, indicating that buckling does not have to be rife to induce formation of shear bands and/or global failure. This confirms past findings on the initiation of failure in dense systems from numerical and experimental data [11,30,36-38].

\section{CONFORMATIONAL STABILITY, TRANSITIONS, AND DYNAMICAL BARRIERS}

At each strain state in the cyclic shear loading history, the assembly along with its constituent particles and mesoscopic particle clusters are each in static equilibrium. Under this condition, we are interested in the ability of a particle cluster to maintain or regain a stable position when acted upon by external forces. With the recently developed techniques from structural mechanics [11] and the ability to measure contact forces $[10,41,46]$, we can now compute the structural stability of the cluster conformation for each particle discussed in Sec. IV, at every equilibrium state of the loading history, and study their transition dynamics. The measure of structural stability in Tordesillas et al. [11], valid for nonconservative, frictional granular assemblies of any size, depends on material properties (i.e., spring stiffnesses and the coefficients of sliding and rolling friction), the contact forces, and the number, positions, and directions of contacts within the cluster. This measure of stability for a nonconservative system is an extension of the ideas proposed by Bagi [42] in studies of jammed states of conservative granular systems. Bagi [42] defined these jammed states to be stable equilibrium states, that is, states in which small load increments result in small displacement increments only and no large or undetermined particle displacements occur. The work in Refs. [11,42] employs essentially the same basic principles that are used in structural mechanics to compute the stability of engineering structures comprising, for example, columns, beams, and arches [55]. We refer readers to Ref. [11] for full details. In what follows, for completeness of the exposition, we first briefly recapitulate the principal elements of the stability measure before presenting our results.

In structural mechanics, the standard procedure for computing the stability of a given structure involves the formulation of a so-called stiffness matrix $K$. This matrix relates the resultant (small) incremental displacements $d \mathbf{u}$ to the applied incremental loads $d \mathbf{q}$ acting on the structure $d \mathbf{q}=\mathbf{K} d \mathbf{u}$. Thus for a granular cluster, the formulation for $K$ involves consideration of the forces which act on each particle in the cluster in question. For the nonconservative cluster conformations 
considered here, the frictional particles experience forces and moments from neighboring particles through their contacts. We assume the particles interact according to classical contact laws: (i) an elastic normal force given by Hooke's law (spring), (ii) an elastic or plastic tangential force given by Hooke's law if elastic and Coulomb's friction law if plastic (spring slider), and (iii) an elastic or plastic contact moment analogous in form to the tangential force [11]. A contact through which elastic forces and moment act is termed elastic; otherwise it is plastic. In general, the formulation of the stiffness matrix for nonconservative systems can be difficult and cumbersome. Consequently, an alternative method which obviates the need to deal with this matrix directly was developed in Ref. [11]. Therein, it was proven that two conservative systems, denoted by $S_{+}$and $S_{-}$, having only elastic contacts, can be constructed for any nonconservative (frictional) granular cluster so that their stabilities provide upper and lower bounds on the cluster stability. $S_{+}$is constructed by replacing every plastic contact in the cluster with a resistive spring of stiffness equal to that used for the elastic contacts in the cluster: $\lambda^{+}$, the stability of $S_{+}$, bounds $\lambda$ from above. $S_{-}$is constructed by replacing every plastic contact in the cluster with a spring of stiffness equal to the lowest value possible (i.e., zero), thereby removing any resistance at that contact: $\lambda^{-}$, the stability of $S_{-}$, bounds $\lambda$ from below. Stiffness matrices $M_{+}$and $M_{-}$are then formulated for $S_{+}$and $S_{-}$, respectively, by relating the small incremental displacements $d \mathbf{u}$ to the applied incremental loads $d \mathbf{q}$ for each system according to the aforementioned relation $d \mathbf{q}=K d \mathbf{u}$. Hence $K=M_{+}$for system $S_{+}$, while $K=M_{-}$for $S_{-}$; in either case, $K$ is independent of $d \mathbf{u}$, and is not necessarily symmetric [42,55]. The minimum eigenvalues of $M_{+}$and $M_{-}$are equal to the stabilities $\lambda^{+}$and $\lambda^{-}$, respectively. The stability of the cluster conformation is expressed as $\lambda=\gamma \lambda^{-}+(1-\gamma) \lambda^{+}$, where $\gamma$ is equal to the ratio of the number of plastic contacts to the total number of contacts in the cluster [11]. $\lambda$ has a dimensional unit of force per unit length, here $N / m$. We emphasize that although the matrices $M_{+}$and $M_{-}$apply to the conservative systems, they each contain information on the frictional granular cluster, that is, the number of contacts and their individual type, position, and orientation; the material properties (i.e., spring stiffness and coefficient of friction); and the boundary and loading conditions. These are all directly measurable quantities in the experiment $[11,41,46]$.

Figure 5 presents the frequency distribution of the structural stabilities computed for the cluster conformations discussed in Sec. IV. It can be observed that pronounced peaks persist throughout the loading history. That such persistent peaks emerge in the stability landscape is surprising, given the

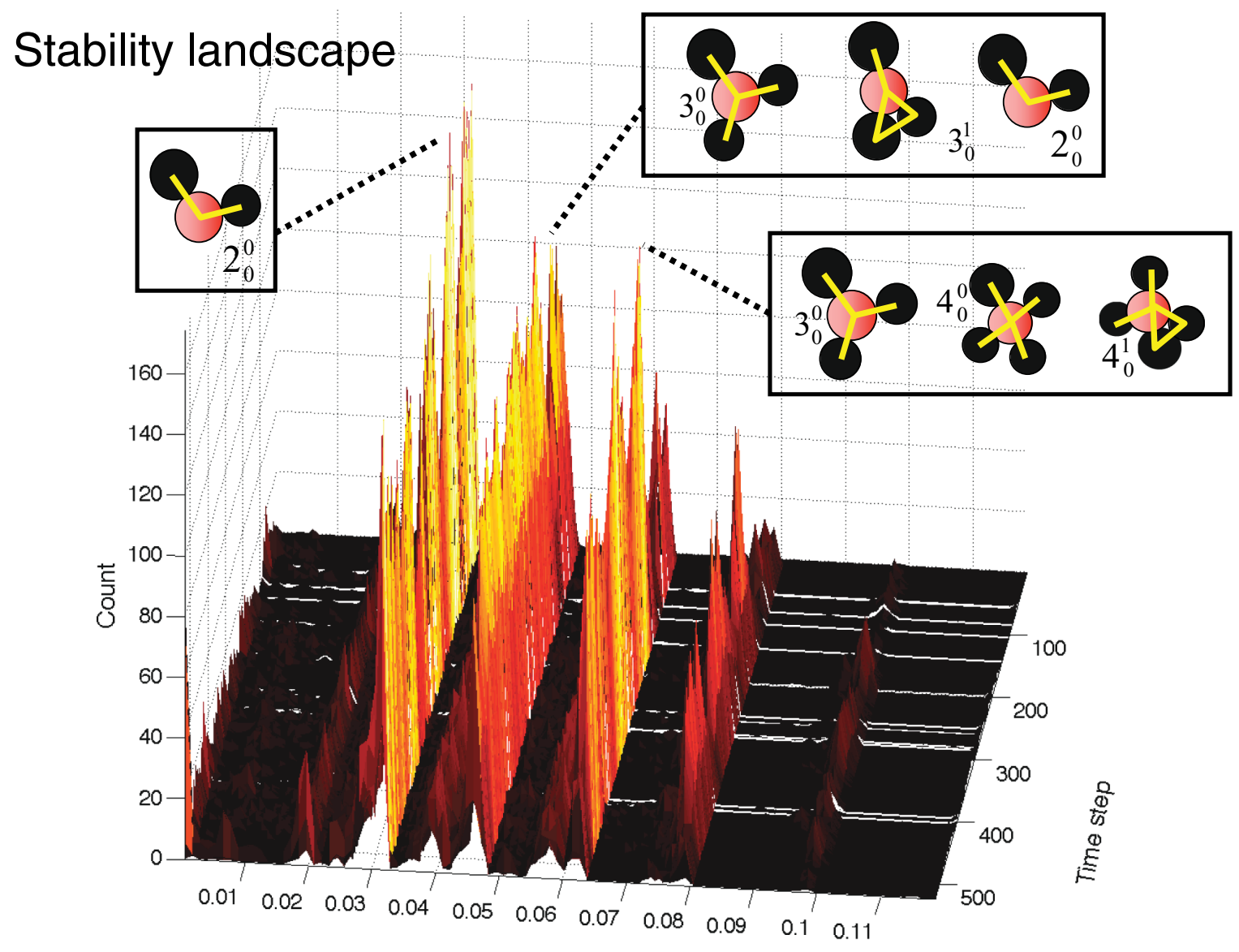

Stability

FIG. 5. (Color online) Stability landscape. The landscape of stability during loading showing the most prevalent granular conformations found in the most resolved stability bands of the cyclic shear experiment. 
extreme heterogeneity in the factors that govern the evolution of stability of a particle relative to its first ring of contacting neighbors. The most dominant conformations and conformational transition sets (sets $1-4$ in Fig. 2, bottom) mainly lie within the stability peaks evident from the well-resolved "bands" in Fig. 5. In particular, the conformations distinguish themselves by increasing stability, which correlates well with an increasing central particle degree: For example, conformations $3_{0}^{0}$ and $3_{0}^{1}$ mainly reside in a lower stability peak than $4_{0}^{0}$ and $4{ }_{0}^{1}$. The peaks in the conformational frequency distribution and the stability landscape are reminiscent of so-called magic numbers encountered in molecular clusters, whose physical rearrangements also govern function through ways in which topology and stability are strongly interrelated [56-58].

We next consider transition dynamics within the stability landscape (Fig. 5). Instead of directly describing transitions between the 28 conformations as a Markov chain, we partition the range of stability values [i.e., $(0,0.1190)$ ] into $\sim 500$ contiguous intervals and model transitions between stability intervals as a Markov chain. To better resolve highly frequented stability values, we use an adaptive partition; that is, the frequency of a conformation possessing a stability value in each interval is roughly equal. We found distinct peaks in stability (Fig. 6, top right) that are consistent with those in Fig. 5. In particular, we find a surprisingly smooth buildup of probability density to each stability peak followed by a precipitous drop.

Sharp changes in the entries of the second largest eigenvector of the stability value transition matrix, as in Fig. 6 (right), delineate dynamical barriers that are infrequently traversed [47]. These sharp changes partition the stability landscape into six major bands (Fig. 6, right): 1, (0,0.0315); 2, (0.0315,0.0397); 3, (0.0397,0.0472); 4, (0.0472,0.0597); $5,(0.0597,0.0630)$; and 6, (0.0630,0.1190). Transitions within each of these six bands are much more likely than transitions between different bands. This banding structure can also be seen in the blocklike structure of the full stability transition matrix (Fig. 6, left).

By aggregating the fine stability intervals into the six large bands, we arrive at a $6 \times 6$ transition matrix describing band-to-band transitions. Typically $40 \%$ to $60 \%$ of stability transitions are within a band. However, we also observe that interband stability transitions prefer to jump over the intervening stability band; that is, transitions among bands 1,3 , and 5 and among bands 2, 4, and 6 are favored (cf. the interlacing of low entries in the block matrix of Fig. 6, left, and the dotted and solid demarcation of the bands in Fig. 6, right). Based on this observation, we can further aggregate by setting state 1 to be the union of bands 1,3 , and 5, and state 2 to be the union of bands 2,4 , and 6 , to produce a $2 \times 2$ transition matrix $B=[0.92150 .0768 ; 0.18720 .8115]$ which effectively partitions the stability space into two almost-invariant stability regions, each consisting of three interlaced stability bands. That is, transitions within state 1 (stability bands $1,3,5$ ) occur with probability 0.9215 and transitions within state 2 (stability bands $2,4,6$ ) occur with probability 0.8115 . On average $70.9 \%$ of clusters are in state 1 and $29.1 \%$ of clusters are in state 2 . The diagonal dominance of $B$ further highlights the "jumping" between every second stability band. Some of the preferred conformations (e.g., $3_{0}^{0}$ ) appear in more than one stability band, thereby indicating that topology does not completely overshadow all other factors that influence stability.

\section{Spectral properties (Aggregated stability bands)}

\section{Transition matrix}

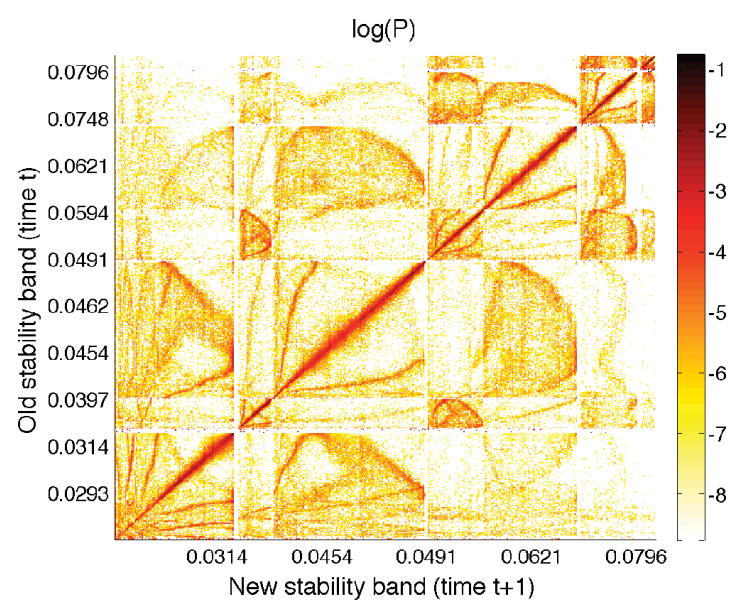

Bands: 12234556
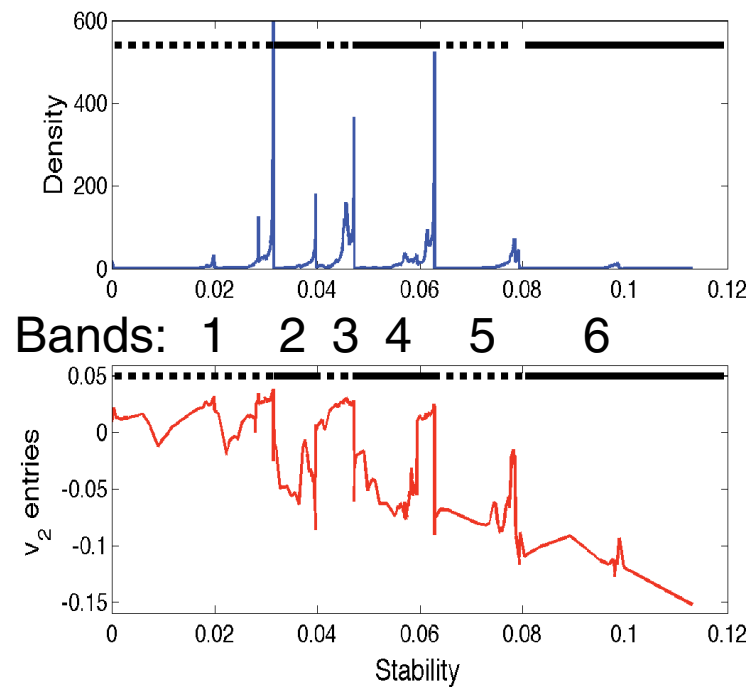

FIG. 6. (Color online) Favored pathways and dynamical barriers in the stability landscape. Scaled entries of the Markov transition matrix reveal the block structure of the stability bands (left). The approximate invariant density of the stability transition matrix revealing the preferred stability peaks (top right). Sudden changes in the entries of the second largest eigenvector correspond to the transition matrix blocks and determine the stability band barriers indicated by alternating solid and dashed lines (bottom right). 


\section{A. Conformational stability transitions during force chain failure by buckling}

We have discussed in Sec. IV the dynamics of force chains, in particular, the continual loss and formation of supporting contacts as the chain responds to and resists an applied force, leading ultimately to buckling. We now continue that line of investigation and turn our attention to the stability transitions of force chain particles during this ultimate failure state of buckling - a mechanism implicated as the precursor for shear band formation and global failure in dense materials [13,17,27, $31,44]$. Conformations of particles in columnar force chains typically possess higher stability (Fig. 7) and those in BFC undergo a similar process of jumping over one stability band (Fig. 7, inset). A relatively higher probability exists for BFC transitions from higher to lower stability states than vice versa. That some force chain particles see an increase in their stability during buckling highlights the system's continual regulation of the topology of contacts and forces to adapt and delay imminent collapse of these load-bearing structures (Figs. 3 and 4). This is entirely consistent with earlier findings on three-force cycles (i.e., three-cycle topology whose contacts each bear above the global average force). These were found to emerge just at the time and location in the sample in greatest need of support: the onset of force chain buckling in what ends up being the shear band [39].

Past experiments and simulations have shown that the buildup of new chains and collapse by buckling of old force chains govern granular deformation in the presence of shear bands [10,11,13,14,21,27,30,38,44]. The "critical state regime" of soil mechanics, in which the material flows at near-steady shear stress and global volume, is believed to

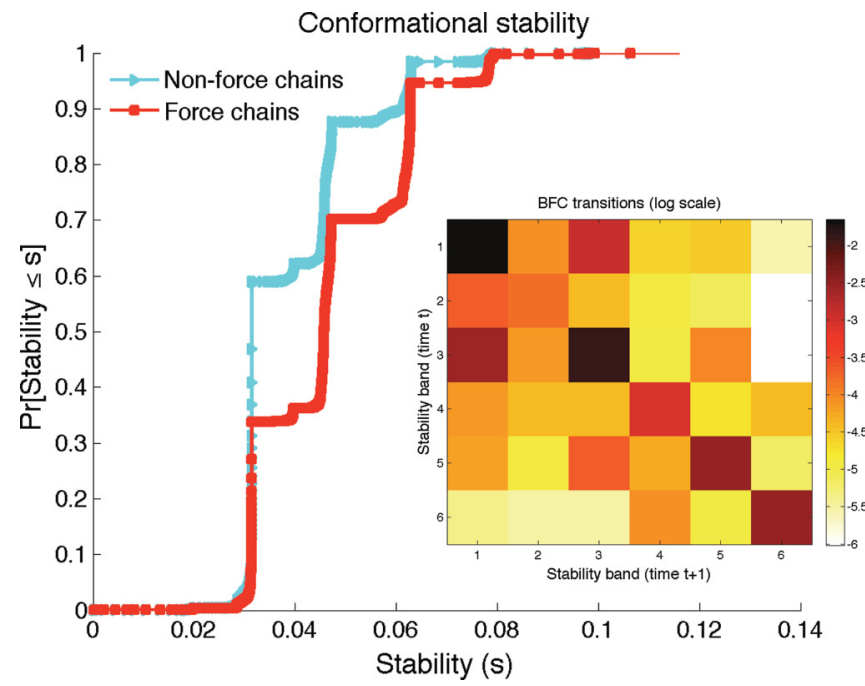

FIG. 7. (Color online) Force chains need lateral support for stability. Cumulative distribution function (CDF) of stability of force chain particle conformations (red square) and non-force-chain conformations (blue triangle). Force chain particle conformations generally possess higher stability. (Inset) The six stability band transition probabilities of BFC particle clusters are typically within the same band (diagonally dominant) or a lower stability band; probabilities below the diagonal are greater than those above the diagonal, demonstrating that buckling is more likely to cause a transition from a higher stability state to a lower stability state.
TABLE I. Dominant cluster (DC) within stability bands and relationship to particles in force chains and buckled force chains. In bands 5 and 6, multiple clusters whose populations are on a par with each other dominate

\begin{tabular}{lccc}
\hline \hline $\begin{array}{l}\text { Stability } \\
\text { band }\end{array}$ & $\begin{array}{c}\text { Dominant } \\
\text { cluster (DC) }\end{array}$ & $\begin{array}{c}\text { DC in force } \\
\text { chain }(\%)\end{array}$ & $\begin{array}{c}\text { Buckled force chain } \\
\text { with DC }(\%)\end{array}$ \\
\hline 1 & $2_{0}^{0}$ & 40 & 68 \\
2 & $2_{0}^{0}$ & 43 & 42 \\
3 & $3_{0}^{0}$ & 65 & 83 \\
4 & $3_{0}^{0}$ & 65 & 54 \\
5 & $4_{0}^{0}, 4_{0}^{1}$ & 79,73 & 54,37 \\
6 & $4_{0}^{0}, 4_{0}^{1}, 5_{0}^{1}$ & $80,76,87$ & $31,16,18$ \\
\hline \hline
\end{tabular}

occur when these two processes balance each other [13,43]. Focusing on the six stability bands, we find the following: The topology of the dominant cluster in the higher stability bands has greater or equal degree relative to the dominant cluster in the lower stability bands; different stability bands can have the same dominant cluster topology; conformations of force chain particles are among the dominant conformations within a stability band; the topology of buckled force chain segments within a stability band follows the dominant conformation but buckling is less likely in higher stability band conformations due to the increased lateral support (see Table I).

\section{LINKING TOPOLOGY, STABILITY, DYNAMICS, AND FUNCTION IN GRANULAR MEDIA}

In summary, the mechanical response of a granular material under load-ultimately rooted in the physical motions or rearrangements of the particles-is systematically and comprehensively quantified here by a study of conformational and stability transition dynamics. We have examined the dynamics of that class of conformations consisting of a particle and its first ring of contacting neighbors in a quasistatically deforming granular material. Relative probabilities of the 28 conformations found, and conformational transitions, have identified the prevalent and persistent rearrangements, including those in key functional structures - the primary load-bearing force chains-which govern the strength and load-carrying capacity of the material. We found self-organization towards favored equilibrium cluster conformations which, in turn, reside within favored stability states in a stability landscape with infrequently traversed dynamical barriers. The most likely conformational transitions during force chain failure by buckling, the mechanism believed to be responsible for global granular failure, correspond to rearrangements among, or loss of, contacts which break the stabilizing trusslike three-cycle topology. We note that the patterns obtained from experimental observations reported here were also found, at least qualitatively, in a discrete element simulation of a two-dimensional polydisperse assembly under monotonic biaxial compression with constant confining pressure.

The granular cluster dynamics uncovered here provides direct structural and mechanical insights into the mechanisms that underpin the rheology of granular media. Conformational transition probabilities comprehensively quantify the relative contributions of the various rearrangements to deformation, 
including those directly responsible for energy dissipation and global failure of the material. This opens the door to robust and tractable predictive continuum models of granular deformation and failure (e.g., [21]). The patterns of self-organization are remarkably reminiscent of those seen in molecular clusters (e.g., $[56,57])$. Anomalously high density stability states, populated by clusters with specific number of contacts in the first ring of neighbors, hint at the possible existence of "magic numbers" in granular systems. In contrast to molecular systems for which the complete tracking of molecular bond formation and breakage is as yet out of reach experimentally [56], granular clusters offer a unique paradigm for studies of emergence and self-organization through conformational transition dynamics: The formation and loss of contacts, as well as the force between constituent particles can be completely tracked in real time, enabling the connections among topology, structural stability, dynamics, and function to be directly unravelled.
Recent studies on nanoscale viral assemblies which found that "concepts borrowed from macroscopic materials science are surprisingly relevant" [58] highlight further common ground and potential synergies in concepts and techniques for unraveling self-organization across a vast range of length scales in complex materials.

\section{ACKNOWLEDGMENTS}

We thank Richard O'Hair for insightful discussions on cluster chemistry and Qun Lin for assistance in generating the data on structural stabilities. This work was supported by the US Army Research Office (W911NF-11-1-0175), the ARC Discovery Project 2012 (DP120104759), and the Melbourne Energy Institute. G.F. is partially supported by the Australian Research Council (DP110100068).
[1] R. O. Ritchie, Nat. Mater. 10, 817 (2011).

[2] D. Weaire, Curr. Opin. Colloid Interface Sci. 13, 171 (2008).

[3] I. Schenker, F. T. Filser, T. Aste, and L. J. Gauckler, J. Eur. Ceram. Soc. 28, 1443 (2008).

[4] Y. Wang, K. Krishan, and M. Dennin, Phys. Rev. E 74, 041405 (2006).

[5] J. Duran, Sands, Powders, and Grains: An Introduction to the Physics of Granular Media (Springer-Verlag, New York, 2000).

[6] M. J. Heinrich, T. Shinbrot, and P. B. Umbanhowar, Proc. Natl. Acad. Sci. USA 97, 12959 (2000).

[7] H. P. Zhu, Z. Y. Zhou, R. Y. Yang, and A. B. Yu, Chem. Eng. Sci. 62, 3378 (2007).

[8] D. Kolymbas and G. Viggiani, eds., Mechanics of Natural Solids (Springer-Verlag, Berlin, Heidelberg, 2009).

[9] D. M. Mueth, Nature (London) 406, 385 (2000).

[10] T. S. Majmudar and R. P. Behringer, Nature (London) 435, 1079 (2005).

[11] A. Tordesillas, Q. Lin, J. Zhang, R. P. Behringer, and J. Shi, J. Mech. Phys. Solids 59, 265 (2011).

[12] A. Ord and B. E. Hobbs, Phil. Trans. R. Soc., A 368, 95 (2010).

[13] A. L. Rechenmacher, J. Mech. Phys. Solids 54, 22 (2006).

[14] F. Radjai, D. E. Wolf, M. Jean, and J.-J. Moreau, Phys. Rev. Lett. 80, 61 (1998).

[15] S. A. Hall, D. M. Wood, E. Ibraim, and G. Viggiani, Granular Matter 12, 1 (2009).

[16] R. Arevalo, I. Zuriguel, and D. Maza, Phys. Rev. E 81, 041302 (2010).

[17] F. Alonso-Marroquin, I. Vardoulakis, H. J. Herrmann, D. Weatherley, and P. Mora, Phys. Rev. E 74, 031306 (2006).

[18] S. J. Antony, Phil. Trans. R. Soc., A 365, 2879 (2007).

[19] N. P. Kruyt and S. J. Antony, Phys. Rev. E 75, 051308 (2007).

[20] A. Lemaitre, Phys. Rev. Lett. 89, 195503 (2002).

[21] D. M. Walker and A. Tordesillas, Phys. Rev. E 85, 011304 (2012).

[22] C. S. Chang, Mech. Mater. 16, 13 (1993).

[23] K. Valanis, Acta Mech. 116, 1 (1996).

[24] D. Kolymbas, ed., in Constitutive Modelling of Granular Materials (Springer-Verlag, Berlin, 2000), pp. 11-24.
[25] Y. F. Jop and O. Pouliquen, Nature (London) 441, 727 (2006).

[26] A. Tordesillas and M. Muthuswamy, Acta Geotech. 3, 225 (2008).

[27] P. A. Cundall and O. D. L. Strack, Geotechnique 29, 47 (1979).

[28] D. Bi, J. Zhang, B. Chakraborty, and R. P. Behringer, Nature (London) 480, 355 (2011).

[29] N. P. Kruyt and L. Rothenburg, J. Stat. Mech. (2006) P07021.

[30] A. Tordesillas and M. Muthuswamy, J. Mech. Phys. Solids 57, 706 (2009).

[31] K. E. Daniels and N. W. Hayman, J. Geophys. Res. 113, B11411 (2008).

[32] P. A. Johnson and X. Jia, Nat. Mater. 6, 99 (2007).

[33] I. Perez and J. Gallego, Constr. Build. Mater. 24, 340 (2010)

[34] F. Vivanco, F. Melo, and C. Fuentes, Int. J. Bifurcat. Chaos 19, 3533 (2009).

[35] D. M. Walker and A. Tordesillas, Int. J. Solids Struct. 47, 624 (2010).

[36] A. Tordesillas, M. Muthuswamy, and S. D. C. Walsh, J. Eng. Mech.-ASCE 134, 1095 (2008).

[37] A. Tordesillas, J. Zhang, and R. P. Behringer, Geomech. Geoeng. 4, 3 (2009).

[38] A. Tordesillas, Philos. Mag. 87, 4987 (2007).

[39] A. Tordesillas, D. M. Walker, and Q. Lin, Phys. Rev. E 81, 011302 (2010).

[40] A. Tordesillas and D. M. Walker, Advances in Bifurcation and Degradation in Geomaterials: Proceedings of the Ninth International Workshop on Bifurcations and Degradations in Geomechanics (Springer, New York, 2011).

[41] J. Zhang, T. S. Majmudar, A. Tordesillas, and R. P. Behringer, Granular Matter 12, 159 (2010).

[42] K. Bagi, Granular Matter 9, 109 (2007).

[43] L. Rothenburg and N. P. Kruyt, Int. J. Solids Struct. 41, 5763 (2004).

[44] M. Oda and H. Kazama, Geotechnique 48, 465 (1998).

[45] H. B. Muhlhaus and I. Vardoulakis, Geotechnique 37, 271 (1987). 
[46] J. Zhang, R. J. Farhadi, R. P. Behringer, T. S. Majmudar, and A. Tordesillas, in Powders and Grains: AIP Conference Proceedings, Vol. 1145 (AIP-Press, Melville, New York, 2009), pp. 553-556.

[47] G. Froyland, Phys. D (Amsterdam, Neth.) 200, 205 (2005).

[48] G. Froyland, K. Padberg, M. H. England, and A. M. Treguier, Phys. Rev. Lett. 98, 224503 (2007).

[49] M. Dellnitz, G. Froyland, C. Horenkamp, and K. Padberg, GAMM-Mitteilungen 32, 47 (2009).

[50] M. Dellnitz, G. Froyland, C. Horenkamp, K. Padberg, and A. S. Gupta, Nonlinear Processes Geophys. 16, 655 (2009).

[51] G. Froyland, N. Santitissadeekorn, and A. Monahan, Chaos 20, 043116 (2010).
[52] N. Santitissadeekorn, G. Froyland, and A. Monahan, Phys. Rev. E 82, 056311 (2010).

[53] G. Froyland, C. Horenkamp, V. Rossi, N. Santitissadeekorn, and A. Sen Gupta, Ocean Modelling 52-53, 69 (2012).

[54] S. H. Strogatz, Nature (London) 410, 268 (2001).

[55] Z. P. Bažant and L. Cedolin, Stability of Structures: Elastic, Inelastic, Fracture, and Damage Theories (Oxford University Press, New York, 1991).

[56] K. Henzler-Wildman and D. Kern, Nature (London) 450, 964 (2007).

[57] R. V. F. Janssens, Nature (London) 435, 897 (2005).

[58] W. H. Roos, R. Bruinsma, and G. J. L. Wuite, Nat. Phys. 6, 733 (2010). 